\title{
Microwave-induced thermoablation with Amica-probe is a safe and reproducible method to treat solid renal masses: Results from a phase I study
}

\author{
RICCARDO BARTOLETTI $^{1}$, ENRICO MELIANI ${ }^{1}$, ALCHIEDE SIMONATO ${ }^{2}$, PAOLO GONTERO ${ }^{3}$, GIOVANNA BERTA $^{3}$, \\ PAOLO DALLA PALMA ${ }^{4}$, ELENA LEONARDI ${ }^{4}$, TOMMASO CAI ${ }^{5}$ and GIORGIO CARMIGNANI ${ }^{2}$ \\ ${ }^{1}$ Department of Urology, Santa Maria Annunziata Hospital, University of Florence, Florence; \\ ${ }^{2}$ Department of Urology, University of Genoa, Genoa; ${ }^{3}$ Department of Urology, University of Turin, \\ Turin; Departments of ${ }^{4}$ Pathology and ${ }^{5}$ Urology, Santa Chiara Hospital, Trento, Italy
}

Received April 27, 2012; Accepted June 8, 2012

DOI: 10.3892/or.2012.1950

\begin{abstract}
Microwave thermal ablation (MWTA) could be considered in the future for treating small solid renal masses. The aim of the present study was to determine both the tolerability of the new Amica-probe applicator-induced MWTA used in vivo on patients with solid renal masses and the effects of heating on renal tumors and normal renal parenchyma. Fourteen patients with renal masses eligible for open radical nephrectomy were enrolled in this phase I study. All patients underwent MWTA of renal masses during the open surgery procedure before clamping of renal vascular pedicle. The effects of MWTA on patients' coagulation and tumor/renal vasculature were investigated. The histological effects of MWTA on the tumor and intralesional vital tumor cell skipping were also evaluated. The MWTA-induced lesion diameters were measured to calculate both the overall ablation volume and the lesion sphericity index (SI). The Clavien-Dindo classification was used. In all patients the RENAL score was 9.4 (8-12) and the Charlson comorbidity index was 4.8 (3-7). MWTA-induced lesion size was $44.14 \mathrm{~mm}( \pm 22.59)$. Mean SI was $1.08( \pm 0.2)$. No significant differences among coagulation clinical parameters were found. No local bleeding after MWTA treatment was reported. According to the ClavienDindo classification, there were two grade II perioperative complications due to the tumor extent but not related with the MWTA treatment. No residual vital tumor cells inside the MWTA-induced lesions were found. Telephone interview at $27.4( \pm 4.2)$ months mean follow-up did not find any long-term adverse events due to previous MWTA treatment. AmicaProbe applicator-induced MWTA is a safe and reproducible method to treat solid renal masses.
\end{abstract}

Correspondence to: Dr Tommaso Cai, Department of Urology, Santa Chiara Hospital, Largo Medaglie d'Oro 9, Trento, Italy E-mail: ktommy@libero.it

Key words: microwave, minimally invasive treatment, kidney neoplasms, thermoablation

\section{Introduction}

Prevalence of small solid renal masses in adult population recently increased probably due to the large number of people that daily undergo preventive diagnostic investigations such as ultrasonography and computed tomography (CT) scan for unrelated symptoms, resulting in incidental tumor detection (1). Patients' comorbidities, such as cardiovascular or respiratory diseases, could represent limiting factors for planning adequate subsequent surgical approaches although conservative laparoscopy surgery is now considered the gold standard treatment, particularly for tumors $<3-4 \mathrm{~cm}$ (2). Minimally invasive procedures, such as cryoablation (CryA) and radiofrequency ablation (RFA), have been recently proposed by different authors as alternative methods of treatment to conservative surgery with satisfactory results in terms of perioperative complications such as hemorrhage episodes or metabolic problems related to prolonged period of general anaesthesia (3). Some doubts remain on the efficacy of these methods on tumor complete eradication due to the high prevalence of tumor local recurrences due to persistent vital tumor cells in the context of the ablated tissue (tumor skipping) (4). Both CryA and RFA are based on the application of physical means (cold and heat respectively) at the centre of the lesion to induce tumor tissue necrosis. Both of them are suitable for clinical application either through laparoscopic trocars or ultrasound/CT-guided percutaneous approaches (5). Microwaves are a source of electromagnetic energy which, similarly to radiofrequency or cryoablation, could be considered in the future for treating small solid renal masses percutaneously or laparoscopically (5). Indeed, with respect to RFA and CryA, microwave thermal ablation (MWTA) allows for a more rapid and homogeneous ablation, due to its reduced sensitivity to local variations of tissue physical properties $(6,7)$. We previously studied the in vivo effects of MWTA on porcine kidney, proving that MWTA is a safe and effective procedure. In particular, we demonstrated the absence of viable cells inside the ablation volume and the absence of any damage to the renal parenchyma outside it (8). Although empirical studies on the effects of MWTA in the treatment of small renal 
masses were previously reported by other authors on small series of patients, none of them reported adequate information on patients tolerability and pathological effects on ablated tissues $(4,5)$. The aim of this phase I prospective multicentre study was to determine both the tolerability of the new Amicaprobe V4 applicator-induced MWTA administered in vivo on patients with solid renal masses and the effects of heating on the renal tumors and normal renal parenchyma.

\section{Materials and methods}

Study design. From February 2009 to June 2010, 14 consecutive patients affected by solid renal masses eligible for open radical surgical treatment were enrolled in three different Italian Urological Centers. We considered for enrolling only patients with renal masses who were candidates to open radical nephrectomy in order to obtain useful adjunctive information on both the anatomical extent and the distribution of MWTA on tumor and peritumoral healthy renal tissues. The patients underwent standard laboratory examinations and radiological evaluations before the treatment. The effects of MWTA on patients' coagulation, tumor/renal parenchyma and tumor skipping were assessed. Patients were informed on the risk of a new therapy with no long-term follow-up data and agreed to participate in the study. The present study was approved by the Research Ethics Committee in each of the Centers participating in the study (IRB Coordinator Center no. URO/01/2008-322/2008). Written informed consent was obtained from all patients before treatment. The study was conducted in line with Good Clinical Practice guidelines and with the Ethical Principles laid down in the latest version of the Declaration of Helsinki.

Inclusion and exclusion criteria. Requirements for inclusion were the presence of a single, solid, contrast-enhanced parenchymal renal mass (attenuation increase, $>15 \mathrm{H}$ on contrast-enhanced CT or $>15 \%$ on gadolinium-enhanced MRI) consistent with renal cell carcinoma on preoperative imaging and to be scheduled for radical nephrectomy through an open approach (9). Patients affected by major concomitant diseases that precluded the surgical treatment, ASA score $\leq 3$, poor performance status (ECOG, 3-4), previous abdominal major surgery, severe medical or psychiatric illness that precluded adequate informed consent, $<18$ or $>85$ years, who had undergone radiation therapy to the retroperitoneum were excluded.

RENAL score and Charlson comorbidity index. The RENAL nephrometry score was calculated in accordance with Kutikov and Uzzo (10). In brief, standardized points (1-3 points/ descriptor) were assigned based on tumor size, endophytic/ exophytic properties, nearness to collecting system and lesion location relative to polar lines. Moreover, the Charlson comorbidity index was calculated by using the software purposed in the website of the Institute for Algorithmic Medicine (Texas non-profit Corporation) (http://www.medal.org/ OnlineCalculators/ch1/ch1.13/ch1.13.01.php) (11).

Microwave probe applicator. The system used for MWTA was HS AMICA, with the Amica-probe V4 applicator (Hospital Services SpA, Aprilia, Italy), which is a technical development

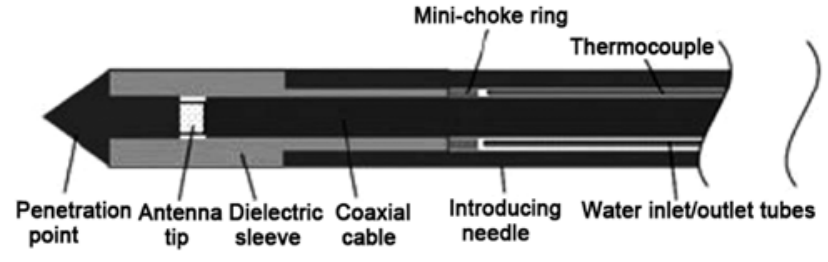

Figure 1. Sagittal section of Amica-probe V4.

of a previously described applicator (Amica-probe V3) (12). Amica-probe V4 includes a coaxial antenna and an integrated hydraulic circuit to cool the coaxial feeding line. The antenna is provided with a patented device (minichoke) for the entrapment of back-propagating waves and is terminated by a sharp point for easy penetration into the tissues (Fig. 1). Control over the coagulative lesion size is obtained by setting the microwave power level and delivery time.

Surgical treatment and MWTA. After the surgical exposure of the kidney and before vascular renal pedicle clamping, a Tru-cut biopsy specimen was taken from the tumor, in order to obtain a tumor sample for histopathological analysis. Then, the Amica-probe V4 applicator was inserted in the bed of Tru-cut needle into the tumor. The applicator was placed at least $3 \mathrm{~cm}$ deep into the tumor, for tumor size $>4-5 \mathrm{~cm}$ (calculated on the basis of CT scan resolution), and at least $1 \mathrm{~cm}$ beyond the tumor, in the case of tumor size $<4 \mathrm{~cm}$. Such different applicator insertion methods were used in order to verify the complete tumor ablation in subjects with tumor size $<4 \mathrm{~cm}$. For each patient, $50 \mathrm{~W}$ microwave power was delivered into the renal tissue for $5 \mathrm{~min}$. The selection of power and time of exposure to MWTA was determined on the basis of previous studies on porcine kidney where these two parameters were standardized to obtain spheroidal shaped repeteable lesions (8). The probe was removed, the renal blood vessels were promptly legated and the surgeon proceeded to classic radical nephrectomy. The surgical specimen, consisting of the kidney and tumor within the Gerota fascia, was removed en bloc and underwent pathological analysis for standard hystopathological diagnosis, microwave thermo-induced effects in the context of both tumor and healthy renal tissue and tumor skipping evaluation.

Heating-induced lesion measurement and histopathological analysis. Pathological samples were collected from the ablated tissues immediately after surgery to investigate neoplastic vital cell skipping. Small tumor samples were collected from the tumor bed after adequate sagittal and horizontal line kidney sections useful for both the tumor and the microwave thermoinduced lesion measurements. These small samples were immediately sectioned and stained with trypan blue according to the procedure described by Allison and Ridolpho (13), in order to test cell viability. Pathologist thus provided for tumor and lesion measurements and tissues were stored in formalin for subsequent staining with haematoxylin and eosin (H\&E) and microscopical analysis. Pathological evaluation was carried out by one dedicated pathologist in each center, in accordance with Bartoletti et al (8). All samples were analyzed to evaluate the effect of microwave energy delivered to renal tissue (no 
Table I. Patient characteristics at baseline.

\begin{tabular}{lcccc}
\hline Characteristics & Mean & SD & Median & Range \\
\hline Age (years) & 70.9 & 9.8 & 70 & $53-88$ \\
Charlson CI & 4.8 & 1.2 & 4 & $4-7$ \\
Lesion diameter (mm) & 47.00 & 18.7 & 44 & $40-92$ \\
RENAL score & 9.4 & 1.1 & 9 & $8-12$ \\
D-dimer (mg/l) & 226.8 & 87 & 225.5 & $102.5-433.3$ \\
INR & 1.01 & 0.08 & 0.95 & $0.89-1.11$ \\
aPTT & 31 & 3.9 & 30.5 & $23-37.5$ \\
\hline
\end{tabular}

Enrolled patients, $\mathrm{n}=14$ (12 males, 2 females). SD, standard deviation; Charlson CI, Charlson comorbidity index.

skipping) and the effect of energy on the collecting system or peritumoral healthy renal tissue, as previously described (8). All ablation lesions were assessed using callipers for both the short-axis diameter (r1, perpendicular to the applicator) and the long-axis diameter (r2, along the applicator). In addition, both diameters were used to calculate the sphericity of the ablation. Sphericity index (SI) was defined as the volume of ablation divided by the volume sphere, using only the greatest diameter. According to Hines-Peralta et al (14), the volume of ablation was defined as the calculated volume of an ellipsoid, obtained by using $\mathrm{r} 1$ and $\mathrm{r} 2$ radii. As suggested by these authors, SI was simplified to $\mathrm{rl}^{2} / \mathrm{r} 2$ (2); a perfect sphere has an index of 1.0 and an ellipsoid $<1.0$ (14).

Trypan blue exclusion test. Trypan blue exclusion test (Life Technologies) is able to accurately determine cell viability. In fact, if cells take-up trypan blue, they are considered nonviable. Live cells or tissues with intact cell membranes are not colored. After the Amica-probe application, a sample of the renal lesion was collected and immediately sent to laboratory in isotonic salt solution under refrigerate conditions. Renal cells were added to a solution $0.4 \%$ of trypan blue in buffered isotonic salt solution, $\mathrm{pH}$ 7.2-7.3, such as phosphate-buffered saline and were examined immediately under a microscope at low magnification. In order to obtain reproducible results, the sample collection and tissue processing was performed according to $\mathrm{Wu}$ et al (15).

Preoperative complication evaluation. The classification of perioperative complications was performed using the ClavienDindo classification (16).

Statistical analysis. All ablation diameters are reported as the mean $( \pm \mathrm{SD})$ and were compared using ANOVA, followed by Bonferroni test, when appropriate, with $\mathrm{P}<0.05$ (two-sided) considered to indicate significance. All statistical calculations were performed with the Statistical Package for Social Sciences 11.0 (SPSS, Inc., Chicago, IL, USA).

For the ablation equipment, materials, support was provided by Hospital Services $\mathrm{SpA}$, and the results were analyzed and discussed independently of any influence of the Sponsor Company.

\section{Results}

Fourteen patients (12 men and 2 women; mean age, 70.9 \pm 9.8 ; range, 53-88 years) underwent open radical nephrectomy. The mean age-adjusted Charlson comorbidity index was 4.8 (range, 3-7). The mean RENAL score was 9.4 (range, 8-12). Table I shows the clinical and laboratory characteristics of enrolled patients.

Clinical and laboratory results. No significant differences between D-dimer, aPPT and INR values were found before and after MWTA and surgery treatment independently from previous anti-platelet medical treatments. Two patients $(14.2 \%)$ with tumor extension to vena cava (pT3b), showed low grade complications (Clavien II) that required blood transfusions. This procedure was, however, independent from the parenchyma MWTA treatment. All patients had a normal postoperative time of hospitalization (mean, 3.1 days; range, 3-5) without relevant complications concerning both the wound healing and the laboratory analyses regularization. No short-term significant bleeding was collected from draining tubes which were removed just before patient discharge. No adverse events due to co-morbidities were observed during hospitalization or after long-term follow-up (27.4 \pm 4.2 months). Noticeable absence or reduction bleeding from the tumor was described by different surgeons overall in patients with smaller tumors, although this parameter could not be scientifically quantifiable.

\section{Histopathological results}

Macroscopic evaluation. MW-induced lesion size was $44.14 \mathrm{~mm}( \pm 22.59)$. All removed kidneys were sectioned along three different planes (sagittal, orizontal and transversal) in order to obtain precise measurement of both tumor and MWTA-induced lesion diameters. Mean SI of MWTAinduced lesions was $1.08( \pm 0.2)$. Fig. 2 shows the distribution of SI values among all patients. All pathological and laboratory findings are presented in Table II. The inner area of the MWTA-induced lesions appeared pale and necrotic, the middle area hyperemic, while a purple border represented the outer layer. H\&E staining showed homogeneous coagulative necrosis at the lesion core (inner area) surrounded by renal cells (Fig. 3A). 
Table II. Pathological and laboratory findings after treatment.

Pathological findings

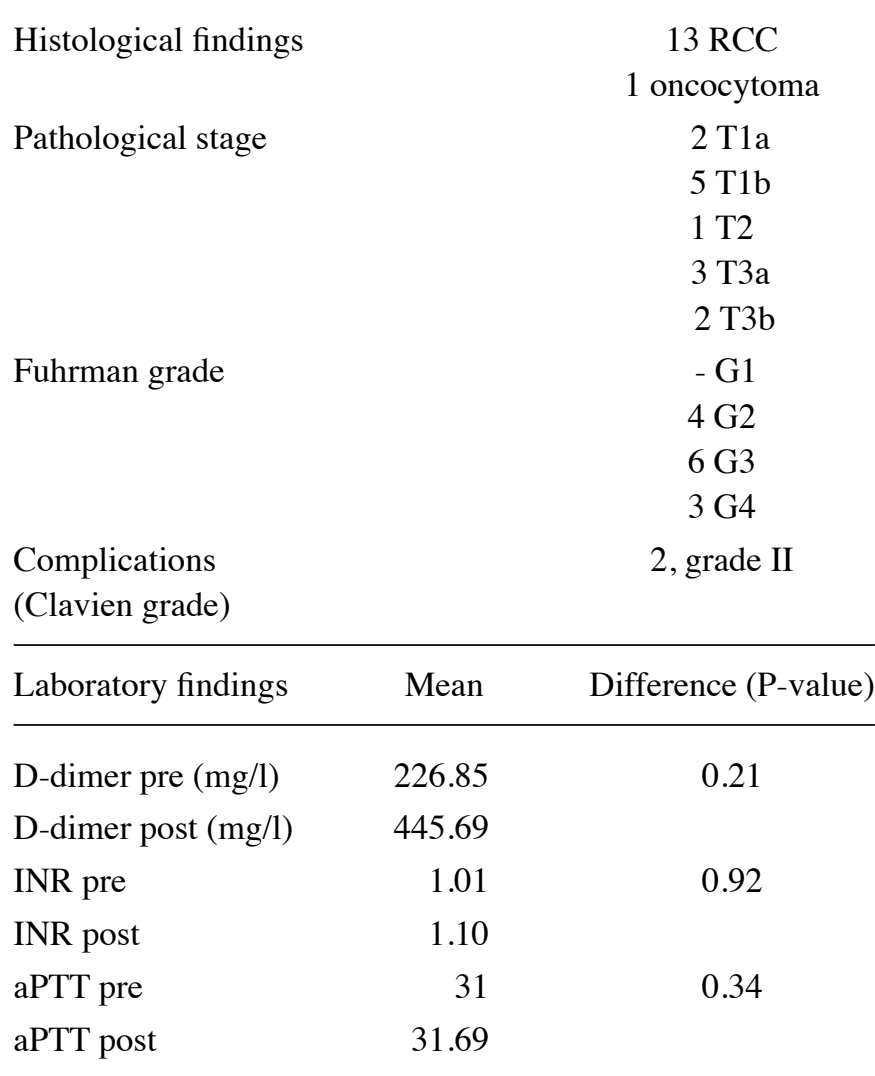

RCC, renal cell carcinoma.

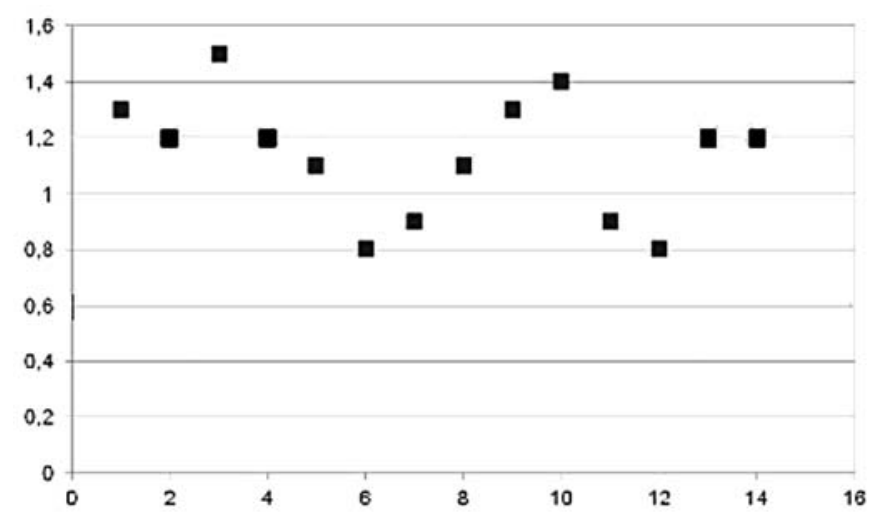

Figure 2. Distribution of sphericity index values among all treated patients.

Microscopic evaluation. Renal cells appear with irreversible cell death, as shown by pyknotic nuclei and nuclear disruption. There were variable amounts of granulation tissue, with immature fibroblasts and inflammatory cells in the boundary region (middle and outer areas). Beyond these, only healthy tissue was found and no skipping was noted in any sample (Fig. 3B). Moreover, histological review of the wide ablation zones showed the inner zone to have uniform cell death by trypan blue exclusion test. No residual vital tumor cells inside the MW-induced lesions were found.

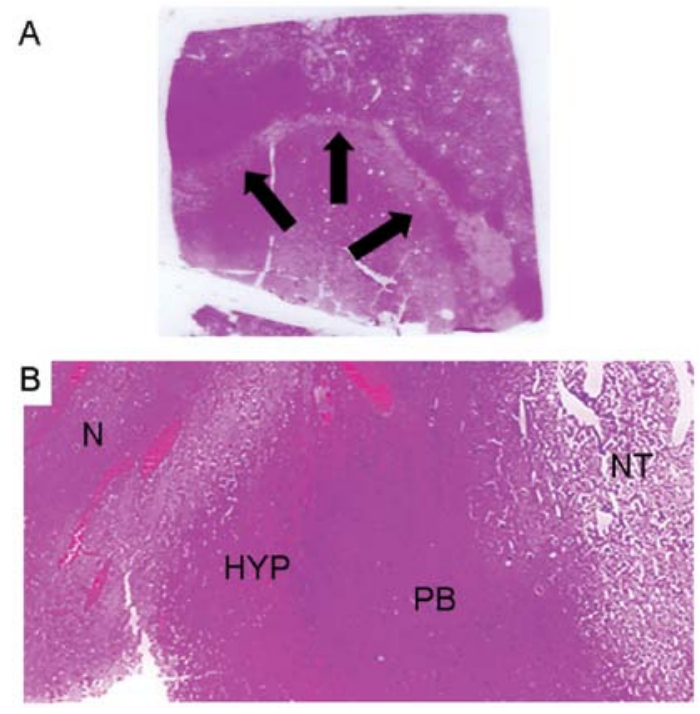

Figure 3. Pathological findings after micro-wave-thermal-ablation (H\&E; magnification, $\mathrm{x} 40$ ). (A) Gross inspection of the ablated kidney tissue. (B) Three areas of ablation were noted when compared to the peripheral normal-looking parenchyma (NT). The inner area appeared pale and necrotic $(\mathrm{N})$, the middle area hyperemic (HYP), while the outer layer is represented by a purple border (PB).

\section{Discussion}

The effects of MWTA on kidney tissue have been recently evaluated and discussed by several authors with conflicting results. Clark et al (9) demonstrated the feasibility of MWTA in 10 patients with large kidney tumors before radical nephrectomy and demonstrated complete tumor cell kill inside the ablated lesions. Liang et al (17) confirmed the efficacy of MWTA on renal tumors by treating 12 patients with small lesions $(<4 \mathrm{~cm}$ in diameter) and no evidence of recurrence after 20 months of follow-up, while Castle et al (18) reported poor oncologic outcomes and significant complication rates in a series of 10 patients with tumor diameter size ranging from 2.0 to $5.5 \mathrm{~cm}$ treated by laparoscopic- or CT-guided percutaneous MWTA. Rational explanations for such similar discrepancies could be easily found by evaluating either the absence of a standardized and reproducible method for microwave administration or the adequate selection of patients. Our previous study on porcine kidney (8) demonstrated that a standardized method of microwave administration could be obtained by using pre-determined independent variables such as power of treatment and time of exposure to energy. The Amica-probe antenna is a new refrigerated probe able to determine approximately spherical lesions with a maximum of $2.7 \pm 0.3 \mathrm{~cm}$ in the animal kidney tissue by an exposure time of $5 \mathrm{~min}$ and $50 \mathrm{~W}$ power (8). The best results could be obtained by using $15 \mathrm{~min}$ of time exposure and $50 \mathrm{~W}$ power with induced-lesion SI $\sim 1.0$ and diameters from 4.1 to $4.2 \mathrm{~cm}( \pm 0.1)$. Moreover, the thermocouple sensors placed $\sim 4 \mathrm{~cm}$ radially far from the Amica-probe antenna point of insertion showed temperature peaks of $41^{\circ} \mathrm{C}$, thus, confirming the safety of this method on healthy renal tissues. These results could explain the importance of patient selection (only patients with small renal masses could be treated) and reliable technology (this refrigerated probe is able to induce a satisfactory SI) to obtain 
reproducible methods of treatment and evaluable results. This is the first study describing the use of Amica-probe V4 microwave applicator on human kidney tumors, which has been successfully tested. Although a subjective variability of MWs induced lesion diameters due to both the applicator insertion depth and the tumor water content, tumors until $2 \mathrm{~cm}$ could be safely treated by using $50 \mathrm{~W}$ power energy and $5 \mathrm{~min}$ of exposure time to MWs. Clinical studies on MWTA of small renal masses and conservative surgery should be then conducted to obtain extensive clinical clarifications and indications on this fascinating technique. Microwaves create a thermal field that is absorbed by surrounding tissues proportionally to the tissue water content (19). Thus, tissues with greater water content absorb more energy and produce heat, while tissues with lower water content produce less heat and propagate microwaves (20). Testing the Amica-probe antenna on both healthy renal tissue and renal tumor tissue, we found no substantial differences of diffusion of MWs by measuring macroscopically MWTAinduced lesion diameters, although no significant variations of SI were found. This could be related to both the cellular type or the presence of necrosis in the context of the tumor. Nevertheless, large tumors usually present necrotic areas in their context while small tumors tends to be more compact and homogeneous except oncocytomas. All patients selected for this study presented large or complicated renal masses (mean RENAL score, 9.4). This kind of selection aimed to obtain an accurate evaluation of MWs diffusion and spread inside the tumor tissue, thus, avoiding the risk of significant variations in heat-induced lesion diameters due to different tissue resistance of tumor pseudo-capsule. Nevertheless, this could justify future studies on MWTA application on small solid renal masses. The potential tropism of MWs to tumor and renal vasculature represented one of the most important parameters investigated in this study. No differences were found in monitoring the patients haemo-coagulative set up before surgery and after short- and long-term follow-up, showing a limited diffusion of MWs through renal tissue independently from the renal pedicle clamping during the MWTA treatment. All patients underwent open radical nephrectomy after MWTA treatment and only $2 / 14$ of them presented intraoperative bleeding with the need of blood-transfusions due to the presence of a tumor thrombus in the vena cava (Clavien II). The incidence of patients requiring blood-transfusions during radical nephrectomy have been reported as ranging from 18 to $44 \%$ in relation to tumor extension and the need of extensive surgery (21). This complication found in our series has not to be considered as relevant and it was not related to the MWTA tumor treatment. No evidence of tumor-cell skipping was found by using vital staining analyisis in all cases. This could demonstrate that local recurrence of disease, previously described by other authors, is probably due to the incomplete ablation of large tumors where the estimation of surgical results have been entrusted to the macroscopically apparent evidence of ablation in case of both laparoscopic- and CT-guided MWs application. The empirical experience of Muto et al (22) confirmed our results demonstrating that MWs application to small renal tumors $(1.3-4.2 \mathrm{~cm})$ provides good results in terms of tumor follow-up and has to be considered as optimal method for haemostasis making laparoscopic tumor enucleation easier and possible without renal pedicle clamping and haemostatic sutures subsequent to tumor removal. However, the present study shows few limitations that should be taken into account during interpretation of the findings. The MWs diffusion and spread through tumor tissue may vary in relation to its liquid content (water and/or necrosis), thus, large tumors could not be considered as the optimal model although our findings demonstrated satisfactory homogeneous results in terms of both heat-induced lesion diameters and SI.

In conclusion, the Amica-probe V4 microwave antenna is able to determine reproducible results in the treatment of small kidney solid tumors. MWTA is a safe method and could be relatively easily employed for future clinical efficacy and comparative studies.

\section{Acknowledgements}

We are grateful to all members of the R\&D Unit of HS Hospital Service SpA (Aprilia, Italy) for their technical assistance and to Professor John Denton for manuscript language revision.

\section{References}

1. Novara G and Ficarra V: Is laparoscopic cryoablation a less invasive and effective procedure to treat small renal masses? Eur Urol 60: 444-447, 2011.

2. Volpe A, Cadeddu JA, Cestari A, et al: Contemporary management of small renal masses. Eur Urol 60: 501-515, 2011.

3. Clements T, Lin YK and Raman JD: Current status of ablative techniques for small renal masses. Expert Rev Anticancer Ther 11: 879-891, 2011.

4. Kunkle DA and Uzzo RG: Cryoablation or radiofrequency ablation of the small renal mass: a meta-analysis. Cancer 113: 2671-2680, 2008.

5. Zagoria RJ and Childs DD: Update on thermal ablation of renal cell carcinoma: oncologic control, technique comparison, renal function preservation, and new modalities. Curr Urol Rep 13: 63-69, 2012.

6. Bhardwaj N, Strickland AD, Ahmad F, Atanesyan L, West K and Lloyd DM: A comparative histological evaluation of the ablations produced by microwave, cryotherapy and radiofrequency in the liver. Pathology 41: 168-172, 2009.

7. Itoh K, Suzuki Y, Miuru M, Tsukigi M, Ichiyanagi O and Sasagawa I: Posterior retroperitoneoscopic partial nephrectomy using microwave tissue coagulator for small renal tumors. J Endourol 16: 367-371, 2002.

8. Bartoletti R, Cai T, Tosoratti N, et al: In vivo microwave-induced porcine kidney thermoablation: results and perspectives from a pilot study of a new probe. BJU Int 106: 1817-1821, 2010.

9. Clark PE, Woodruff RD, Zagoria RJ and Hall MC: Microwave ablation of renal parenchymal tumors before nephrectomy: phase I study. AJR Am J Roentgenol 188: 1212-1214, 2007.

10. Kutikov A and Uzzo RG: The R.E.N.A.L. nephrometry score: a comprehensive standardized system for quantitating renal tumor size, location and depth. J Urol 182: 844-853, 2009.

11. Charlson ME, Pompei P, Ales KL and MacKenzie CR: A new method of classifying prognostic comorbidity in longitudinal studies: development and validation. J Chronic Dis 40: 373-383, 1987.

12. Bartoletti R, Cai T, Tinacci G, et al: Transperineal microwave thermoablation in patients with obstructive benign prostatic hyperplasia: a phase I clinical study with a new mini-choked microwave applicator. J Endourol 22: 1509-1517, 2008.

13. Allison DC and Ridolpho P: Use of a trypan blue assay to measure the deoxyribonucleic acid content and radioactive labeling of viable cells. J Histochem Cytochem 28: 700-703, 1980.

14. Hines-Peralta AU, Pirani N, Clegg P, et al: Microwave ablation: results with a $2.45-\mathrm{GHz}$ applicator in ex vivo bovine and in vivo porcine liver. Radiology 239: 94-102, 2006.

15. Wu XX, Kakehi Y, Jin XH, Inui M and Sugimoto M: Induction of apoptosis in human renal cell carcinoma cells by vitamin $\mathrm{E}$ succinate in caspase-independent manner. Urology 73: 193-199, 2009. 
16. Clavien PA, Barkun J, de Oliveira ML, et al: The Clavien-Dindo classification of surgical complications: five-year experience. Ann Surg 250: 187-196, 2009.

17. Liang P, Wang Y, Zhang D, Yu X, Gao Y and Ni X: Ultrasound guided percutaneous microwave ablation for small renal cancer: initial experience. J Urol 180: 844-848, 2008.

18. Castle SM, Salas N and Leveillee RJ: Initial experience using microwave ablation therapy for renal tumor treatment: 18 -month follow-up. Urology 77: 792-797, 2011.

19. Carrafiello $\mathrm{G}$, Laganà $\mathrm{D}$, Ianniello $\mathrm{A}$, et al: Percutaneous radiofrequency thermal ablation of renal cell carcinoma: is it possible a day-hospital treatment? Int J Surg 6: 31-35, 2008.
20. Carey RI and Leveillee RJ: First prize: direct real-time temperature monitoring for laparoscopic and CT-guided radiofrequency ablation of renal tumors between 3 and $5 \mathrm{~cm}$. J Endourol 21: 807-813, 2007.

21. Shvarts O, Tsui KH, Smith RB, Kernion JB and Belldegrun A: Blood loss and the need for transfusion in patients who undergo partial or radical nephrectomy for renal cell carcinoma. J Urol 164: 1160-1163, 2000

22. Muto G, Castelli E, Migliari R, D'Urso L, Coppola P and Collura D: Laparoscopic microwave ablation and enucleation of small renal masses: preliminary experience. Eur Urol 60: 173-176, 2011. 